\title{
ON RELAXATION OF NORMALITY IN THE FUGLEDE-PUTNAM THEOREM
}

\author{
TAKAYUKI FURUTA ${ }^{1}$
}

\begin{abstract}
An operator means a bounded linear operator on a complex Hilbert space. The familiar Fuglede-Putnam theorem asserts that if $A$ and $B$ are normal operators and if $X$ is an operator such that $A X=X B$, then $A^{*} X=X B^{*}$. We shall relax the normality in the hypotheses on $A$ and $B$.

THEOREM 1. If $A$ and $B^{*}$ are subnormal and if $X$ is an operator such that $A X=X B$, then $A^{*} X=X B^{*}$.

THEOREM 2. Suppose $A, B, X$ are operators in the Hilbert space $H$ such that $A X=X B$. Assume also that $X$ is an operator of Hilbert-Schmidt class. Then $A^{*} X=X B^{*}$ under any one of the following hypotheses:

(i) $A$ is $k$-quasihyponormal and $B^{*}$ is invertible hyponormal,

(ii) $A$ is quasihyponormal and $B^{*}$ is invertible hyponormal,

(iii) $A$ is nilpotent and $B^{*}$ is invertible hyponormal.
\end{abstract}

1. In this paper an operator means a bounded linear operator on a complex Hilbert space. An operator $T$ is called quasinormal if $T$ commutes with $T^{*} T$, subnormal if $T$ has a normal extension and hyponormal if $\left[T^{*}, T\right]>0$ where $[S, T]=S T-T S$. The inclusion relation of the classes of nonnormal operators listed above is as follows:

\section{Normal $\varsubsetneqq$ Quasinormal $\varsubsetneqq$ Subnormal $\varsubsetneqq$ Hyponormal;}

the above inclusions are all proper [3, Problem 160, p. 101].

The familiar Fuglede-Putnam theorem is as follows ([3, Problem 152], [4]).

TheOREM A. If $A$ and $B$ are normal operators and if $X$ is an operator such that $A X=X B$, then $A^{*} X=X B^{*}$.

In this paper we relax the normality in the hypotheses on $A$ and $B$ in Theorem A, namely, we show that the normality can be replaced by the subnormality of $A$ and $B^{*}$. We show some applications of this result. Finally we present some remarks on Berberian's results [1] which are related to the relaxation of normality in the hypothesis in Theorem A.

2. First we show Theorem 1 as an extension of Theorem A by an easy calculation.

Received by the editors May 16, 1978 and, in revised form, June 16, 1978.

AMS (MOS) subject classifications (1970). Primary 47B20, 47B15; Secondary 47A20, 47B10.

Key words and phrases. Quasinormal operators, subnormal operators, hyponormal operators, quasihyponormal operators, Hilbert-Schmidt operators.

${ }^{1}$ Research supported by Kakenhi 364038. 
THEOREM 1. If $A$ and $B^{*}$ are subnormal and if $X$ is an operator such that $A X=X B$, then $A^{*} X=X B^{*}$.

Proof. A normal extension $N_{A}$ of $A$ on Hilbert space $H$ is given by

$$
N_{A}=\left(\begin{array}{cc}
A & A_{12} \\
0 & A_{22}
\end{array}\right)
$$

acting on a large Hilbert space $K_{A}$ containing $H$ and a normal one $N_{B^{*}}$ of $B^{*}$ on $H$ is also given by

$$
N_{B^{*}}=\left(\begin{array}{cc}
B^{*} & B_{12} \\
0 & B_{22}
\end{array}\right)
$$

acting on another large Hilbert space $K_{B^{*}}$ containing $H$ respectively. We consider an operator matrix

$$
\left(\begin{array}{cc}
B_{22}^{*} & B_{12}^{*} \\
0 & B
\end{array}\right)
$$

acting on $\left(K_{B^{*}} \ominus H\right) \oplus H$. According to the normality of $N_{B^{*}}$, this operator matrix turns out to be normal. For $X$ acting on $H$, we consider $\hat{A}$ and $\hat{X}$ acting on a larger space $H \oplus\left(K_{A} \ominus H\right) \oplus\left(K_{B^{*}} \ominus H\right) \oplus H$ as follows;

$$
\hat{A}=\left(\begin{array}{cccc}
A & A_{12} & 0 & 0 \\
0 & A_{22} & 0 & 0 \\
0 & 0 & B_{22}^{*} & B_{12}^{*} \\
0 & 0 & 0 & B
\end{array}\right), \quad \hat{X}=\left(\begin{array}{cccc}
0 & 0 & 0 & X \\
0 & 0 & 0 & 0 \\
0 & 0 & 0 & 0 \\
0 & 0 & 0 & 0
\end{array}\right)
$$

Clearly $\hat{A}$ is normal and $\hat{A} \hat{X}=\hat{X} \hat{A}$ by the hypothesis $A X=X B$, so that $\hat{A}^{*} \hat{X}=\hat{X} \hat{A}^{*}$ by Theorem $\mathrm{A}$, and comparing the corresponding entries, we have $A^{*} X=X B^{*}, A_{12}^{*} X=0$ and $X B_{12}=0$ so the proof is complete.

REMARK 1. The argument stated above extends readily to cover the case that operators $A$ and $B$ are possibly unbounded, namely, if $A$ and $B^{*}$ are subnormal and if $X$ is a bounded operator such that $A X \supset X B$, then $A^{*} X \supset X B^{*}$.

An operator $T$ is called co-subnormal if $T^{*}$ is subnormal. For normal operators similarity implies unitary equivalence [3, Corollary of Problem 152] and the following corollary is an extension of this result.

Corollary 1. If $A$ is subnormal, $B$ is co-subnormal and they are similar, then they are both normal and unitarily equivalent.

Proof. Although the proof for two normal operators can be modified so as to imply this generalized result, we cite this proof for the sake of completeness. If $X=U P$ is the polar decomposition of the invertible operator $X$, then $U$ is unitary and $P$ is the invertible positive square root of $X^{*} X$. According to the hypotheses that $A$ and $B^{*}$ are both subnormal and $A X=X B$, then $B X^{*}=X^{*} A$ by scrutinizing the proof of Theorem 1 and $X^{*} X B=X^{*} A X=$ $B X^{*} X$, so that $P^{2} B=B P^{2}$, whence $P B=B P$. This result and the hypothesis 
$A U P=U P B$ yield $A U=U B$. Moreover tracing the proof of Theorem 1, $A_{12}^{*} X=0$ and $X B_{12}=0$. As $X$ is invertible by the hypothesis, we have $A_{12}=0$ and $B_{12}=0$, that is, $A$ and $B$ are both normal, so the proof is complete.

Remark 2. With respect to Corollary 1 we remark that there exist two subnormal operators that are similar but not unitarily equivalent $[3$, Solution 156]. In fact Theorem 1 and Corollary 1 do not need symmetric hypotheses on $A$ and $B$, but they need symmetric hypotheses on $A$ and $B^{*}$. In view of this point, it is natural and reasonable to understand that the hypothesis of normality of $A$ and $B$ is considered as that of normality of $A$ and $B^{*}$ in Theorem A.

It is natural to ask whether the subnormality can be replaced by the hyponormality in Theorem 1. But this is an open problem; a modest result is cited in [5, Proposition]. The following result is in this direction.

Corollary 2. If $A$ and $B^{*}$ are hyponormal operators satisfying $S_{A} A=A^{*} S_{A}$ and $S_{B^{*}} B^{*}=B S_{B^{*}}$ and if $X$ is an operator such that $A X=X B$, then $A^{*} X=$ $X B^{*}$, where $S_{A}$ and $S_{B^{*}}$ denote the normal operators such that $\left|S_{A}\right|^{2}=\left[A^{*}, A\right]$ and $\left|S_{B^{*}}\right|^{2}=\left[B, B^{*}\right]$, respectively.

Proof. The hypotheses imply that $A$ and $B^{*}$ are subnormal by [2, Theorem 3] and the desired conclusion follows from Theorem 1.

3. In [1] S. K. Berberian relaxes the hypotheses on $A$ and $B$ in Theorem $\mathrm{A}$ at the cost of requiring $X$ to be of Hilbert-Schmidt class as follows:

Theorem B. Suppose $A, B, X$ are operators in the Hilbert space $H$ such that $A X=X B$. Assume also that $X$ is an operator of Hilbert-Schmidt class. Then $A^{*} X=X B^{*}$ under either of the following hypotheses:

(i) $A$ and $B^{*}$ are hyponormal,

(ii) $B$ is invertible and $\|A\| \cdot\left\|B^{-1}\right\| \leqslant 1$.

In this section we also relax the hypothesis on $A$ in Theorem $B$ at the cost of requiring that $B^{*}$ be invertible hyponormal; that is, if we assume the stronger hypothesis on $B^{*}$, then we may assume the weaker hypothesis on $A$.

An operator $T$ is called quasihyponormal if $T$ satisfies $\left\|T^{2} x\right\| \geq\left\|T^{*} T x\right\|$ for all $x$ in $H$, that is equivalent to $T^{*}\left(T^{*} T-T T^{*}\right) T>0$. It is easily known that the class of quasihyponormal operators properly contains that of hyponormals and that an invertible quasihyponormal is always hyponormal. It is also known that quasihyponormal is not always transaloid, namely, $T-\lambda$ is not always normaloid for any complex $\lambda$. An operator $T$ is also called $k$-quasihyponormal if $T$ satisfies $\left\|T^{k+1} x\right\| \geqslant\left\|T^{*} T^{k} x\right\|$ for all $x$ in $H$, that is, $T^{* k}\left(T^{*} T-T T^{*}\right) T^{k} \geqslant 0$. It is also known that the class of $k$-quasihyponormal operators properly contains the three classes of $k^{\prime}$-quasihyponormal $\left(k^{\prime}<k\right)$, quasihyponormal and nilpotent operators with index $k$.

We show the following theorem as a parallel result to Theorem B. 
Theorem 2. Suppose $A, B, X$ are operators in the Hilbert space $H$ such that $A X=X B$. Assume also that $X$ is an operator of Hilbert-Schmidt class. Then $A^{*} X=X B^{*}$ under any one of the following hypotheses:

(i) $A$ is $k$-quasihyponormal and $B^{*}$ is invertible hyponormal,

(ii) $A$ is quasihyponormal and $B^{*}$ is invertible hyponormal,

(iii) $A$ is nilpotent and $B^{*}$ is invertible hyponormal.

The proof of Theorem 2 is merely modification of Berberian's technique. Notations used here generally conform with [1]. Hilbert-Schmidt class operators form an ideal $\mathcal{H}$ in the algebra $\mathcal{L}(H)$ of all bounded operators on $H$. In [1] an operator $\mathcal{T} \in \mathcal{L}(\mathcal{H})$ is defined by $\mathcal{T} Y=A Y B$ and $\mathcal{T}^{*} Y=A^{*} Y B^{*}$ is shown.

LEMMA 1. If $A$ and $B^{*}$ are $k$-quasihyponormal, then the operator $\mathcal{T}$ in $\mathcal{H}$ defined by $\mathcal{T} Y=A Y B$ is also $k$-quasihyponormal.

Proof. According to the hypotheses of $k$-quasihyponormality of $A$ and $B^{*}$, we have

$$
\begin{gathered}
\left(\mathcal{T}^{* k+1} \mathcal{J}^{k+1}-\mathcal{T}^{* k} \mathcal{T} \mathcal{T}^{*} \mathcal{J}^{k}\right) Y=\left(A^{* k+1} A^{k+1}-A^{* k} A A^{*} A^{k}\right) Y B^{k+1} B^{* k+1} \\
+\left(A^{*} A^{k}\right)^{*} A^{*} A^{k} Y\left(B^{k+1} B^{* k+1}-B^{k} B^{*} B B^{* k}\right)
\end{gathered}
$$

this shows that $\mathcal{T}$ is also $k$-quasihyponormal.

Proof of Theorem 2. Assume (i). Let $\mathcal{T}$ be the operator in $\mathcal{H}$ defined by $\mathcal{T} Y=A Y B^{-1}$. Since $\left(B^{-1}\right)^{*}=\left(B^{*}\right)^{-1}$ is hyponormal and every hyponormal is always $k$-quasihyponormal, $\mathcal{T}$ is also $k$-quasihyponormal by Lemma 1 . Hypothesis $A X=X B$ implies $\mathcal{T} X=X$ and this relation yields $\left\|\mathcal{T}^{*} X\right\|<$ $\|X\|$ by $k$-quasihyponormality of $\mathcal{T}$, so that

$$
\left\|\mathcal{J}^{*} X-X\right\|^{2} \leqslant\|X\|^{2}-2\|X\|^{2}+\|X\|^{2}=0 ;
$$

this implies $\mathcal{T}^{*} X=X$, namely, $A^{*} X\left(B^{-1}\right)^{*}=X$, hence $A^{*} X=X B^{*}$ which is the desired relation. (ii) and (iii) are both special cases of (i) since quasihyponormal and nilpotent operators with index $k$ are also $k$-quasihyponormal, so the proof is complete.

RemarK 3. If $T$ is $k$-quasihyponormal and $\lambda$ is a nonzero proper value of $T$, then $\lambda^{*}$ is a proper value of $T^{*}$ by a similar verification to the one in the proof of Theorem 2 .

ADDENDUM. If $A$ is a subnormal operator on $H$, then there exists a normal operator $N_{A}$ on the Hilbert space $H \oplus H$ whose restriction to $H \oplus\{0\}$ is $A$ (P. R. Halmos, J. Reine Angew Math. 208 (1961), 102-112). In the proof of Theorem 1 we can take $K_{A}=H \oplus H, K_{B^{*}}=H \oplus H$, etc. and all operator matrix entries in sight are operators in $H$ by this remark. We are indebted to Professor S. K. Berberian for this comment.

AdDed IN PROOF. ANOTHER Proof OF TheOREM 1. Considering $N_{A}, N_{B^{*}}$ on $H \oplus H$ as stated in Addendum and also taking $\hat{X}=\left(\begin{array}{ll}X & 9 \\ 0 & 0\end{array}\right)$ on $H \oplus H$, then 
the hypothesis $A X=X B$ implies $N_{A} \hat{X}=\hat{X} N_{B^{*}}^{*}$. Hence $N_{A}^{*} \hat{X}=\hat{X} N_{B^{*}}$ holds by Theorem $A$ and this relation alternatively yields the desired one $A^{*} X=$ $X B^{*}$ by comparing the corresponding entries.

\section{REFERENCES}

1. S. K. Berberian, Extensions of a theorem of Fuglede and Putnam, Proc. Amer. Math. Soc. 71 (1978), 113-114.

2. T. Furuta, K. Matsumoto and N. Moriya, A simple condition on hyponormal operators implying subnormality, Math. Japon. 21 (1976), 399-400.

3. P. R. Halmos, A Hilbert space problem book, Van Nostrand, Princeton, N. J., 1967.

4. C. R. Putnam, On normal operator in Hilbert space, Amer. J. Math. 73 (1951), 357-362.

5. J. G. Stampfli and B. Wadhwa, An asymmetric Putnam-Fuglede theorem for dominant operators, Indiana Univ. Math. J. 25 (1976), 359-365.

Department of Mathematics, Faculty of Science, Hirosaki University, Bunkyo-Cho 3, HIROSAKI, 036 AOMORI, JAPAN 